\title{
Primary cutaneous infection due to Microascus cirrosus: a case report
}

\author{
Lujuan Gao, Junjun Chen, Di Gao and Ming Li
}

\begin{abstract}
Background: Microascus cirrosus, the teleomorph of Scopulariopsis spp., is a saprobic species with a worldwide distribution and rarely causes human infection. In the present paper, we present the first case of primary cutaneous $M$. cirrosus infection in a Chinese female.

Case presentation: A 17-year-old female presented with tender ulceration on her left ankle for three months. Histology revealed multiple branching, septate hyphae and moniliform fungal elements in the dermis. Tissue culture grew $M$. cirrosus, the teleomorph of Scopulariopsis spp., characterized by intercalary and ballooned, chlamydospore-like structures, annellidic and ampulliform conidiogenous cells along with truncated, bullet-shaped, smooth conidia and globose perithecial ascomata with cylindrical necks. Further molecular sequencing confirmed the identification. A diagnosis of primary cutaneous infection due to M. cirrosus was made. Treatment with itraconazole $200 \mathrm{mg}$ per day for 10 weeks achieved significant improvement of the skin lesions.

Conclusions: This case of uncommon mycotic cutaneous infection highlights the importance of mycological examination that help to recognize rare pathogenic fungi.
\end{abstract}

Keywords: Cutaneous, Microascus cirrosus, Itraconazole, Scopulariopsis

\section{Background}

The genera Microascus and Scopulariopsis comprise species that commonly isolated from soil, air, decaying plant material, dung and moist indoor environments [1]. Previously, Scopulariopsis and Microascus species have not been considered as common human pathogenic fungi. However, recently the number of cases caused by these organisms has been on the rise. Among the genera Microascus and Scopulariopsis, M. cirrosus is an uncommon opportunistic fungi, which has been reported to cause superficial infections of toe nail, and systemic infection [2-5]. To our knowledge, primary cutaneous infection due to $M$. cirrosus has not been reported yet. Here, we report the first case of primary cutaneous infection of $M$. cirrosus that responded well to oral itraconazole.

\section{Case presentation}

A 17-year-old otherwise healthy Chinese female presented with tender skin lesion on her left ankle for almost three months. The lesion initially presented as an

\footnotetext{
* Correspondence: zspfklm@163.com

Department of Dermatology, Zhongshan Hospital Fudan University, No.180 Fenglin Road, Xuhui District, Shanghai 200032, China
}

indurated erythema with central dusky necrosis, mimicking insect bites (Fig. 1a). She didn't recall any evident history of trauma. During the same period, she developed extensive petechiae on both lower extremities and was diagnosed as Henoch-Sch nlein purpura in local hospital. Subsequently she was treated with systemic corticosteroids (maximum dosage $30 \mathrm{mg} / \mathrm{d}$ ) for two months. While the petechiae subsided, the ankle erythema ulcerated with suppurative discharge. She was then admitted to our hospital in March, 2017. Physical examination revealed a $3.5 \mathrm{~cm} * 2.5 \mathrm{~cm}$ demarcated ellipsoidal ulceration covered with thick black crust and purulent discharge (Fig.1b). Regional lymph nodes were not palpable. Except for these lesions, the girl was generally in good health.

Histological examination of biopsied tissue revealed multiple branching, septate hyphae and moniliform fungal elements in the dermis (Fig. 2a), which were positive with periodic acid-Schiff and Gomori-Grocott methenamine silver staining (Fig. 2b).

Tissue culture was performed and incubated at both $26^{\circ} \mathrm{C}$ and $35^{\circ} \mathrm{C}$ on Sabouraud's dextrose agar (SDA) and yielded restricted, white to grey and velvety colony at 4 

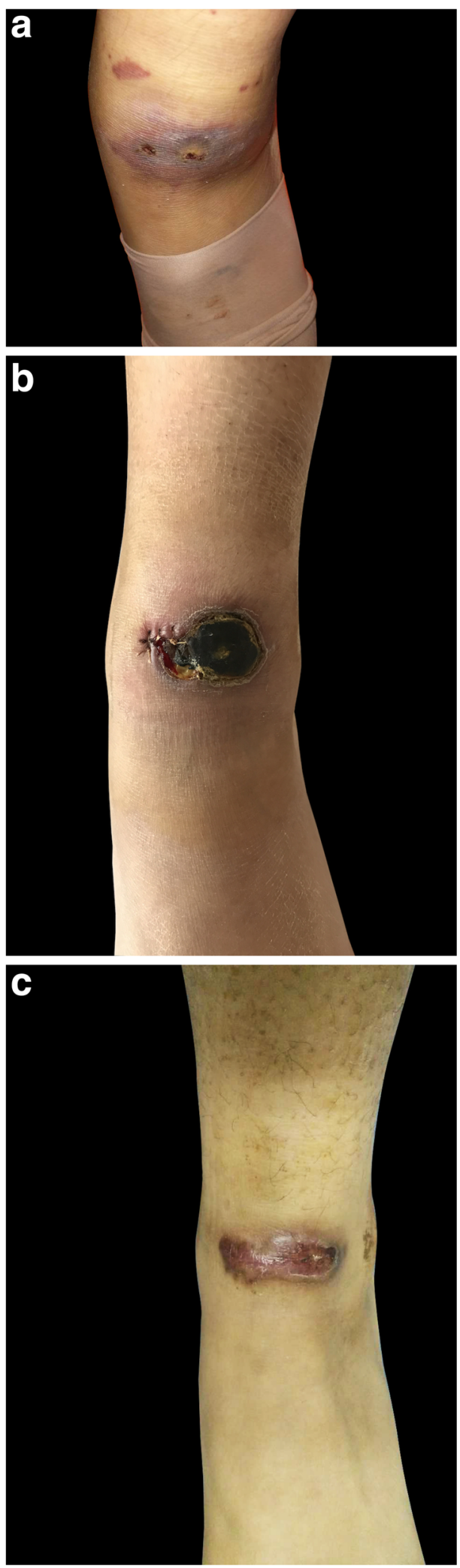

Fig. 1 Clinical appearance. a Indurated erythema with central dusky necrosis at early stage. $\mathbf{b}$ Demarcated ulceration of $3.5 \mathrm{~cm} * 2.5 \mathrm{~cm}$, covered with thick black crust and purulent discharge. c Complete resolution of the ulceration with residual scar

weeks (Fig. 3a). With extended incubation, the colony turned brownish at 8 weeks (Fig. 3b). Slides culture revealed filamentous and septate hyphae with intercalary and ballooned, chlamydospore-like structures, as well as annellidic and ampulliform conidiogenous cells along with truncated, bullet-shaped, smooth conidia (Fig. 3c), which resembled those found in Scopulariopsis species. Within 5 weeks, small black fruiting structures were growing on the surface of the colony, resulting in observance of globose perithecial ascomata with cylindrical necks, and a dark peridium (Fig. 3d). Fungal culture was identified as $M$. cirrosus based on the morphological features and confirmed by the molecular sequencing of internal transcribed spacer (ITS) region gene and $\beta$-tubulin gene. Comparison of the ITS (642 bp) (Genbank accession

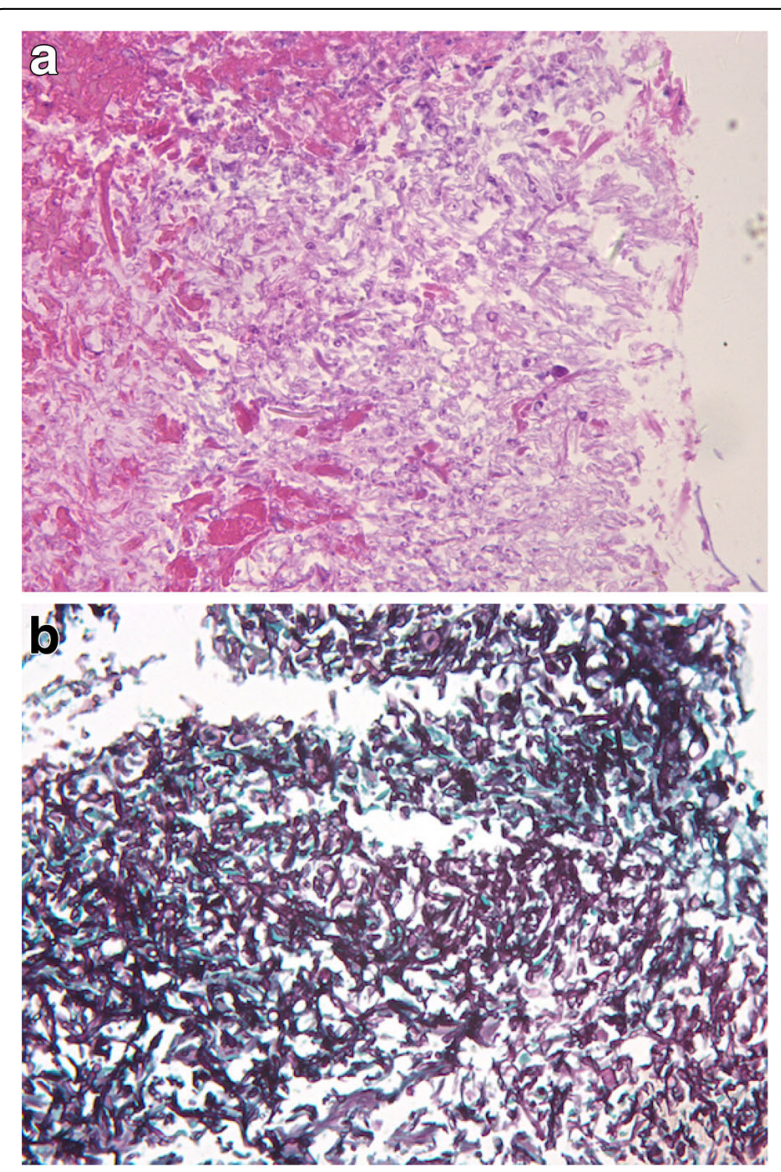

Fig. 2 Histopathology findings. a Hematoxylin-eosin stained specimen revealed multiple branching, septate hyphae and moniliform fungal elements in the dermis (original magnification $\times$ 400). b Gomori-Grocott methenamine silver stain was positive (original magnification $\times$ 400) 

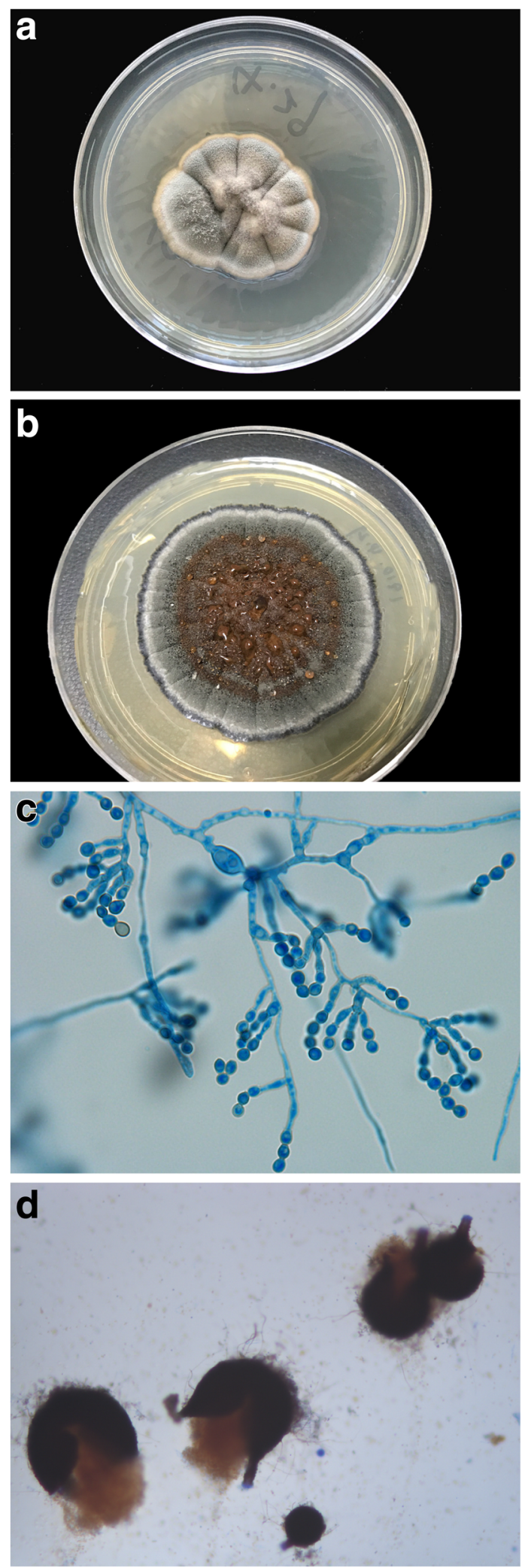

Fig. 3 Mycological findings. ab Fungal culture incubated at $26^{\circ} \mathrm{C}$ on Sabouraud's dextrose agar grew white to grey and velvety colony at 4 weeks and turned brown at 8 weeks. c Lactophenol cotton blue stain of slides culture revealed filamentous and septate hyphae with intercalary and ballooned, chlamydospore-like structures, as well as annellidic and ampulliform conidiogenous cells along with truncated, bullet-shaped, smooth conidia (original magnification $\times 1000$ ). $\mathbf{d}$ Black fruits under microscopy showed globose perithecium with cylindrical necks, and a dark peridium, which are composed of dark brown cells (original magnification $\times 200$ )

number MF450505) and $\beta$-tubulin (501 bp) (Genbank accession number MF455507) sequence with the Genbank database revealed $100 \%$ similarity with $M$. cirrosus reference strain CBS 116405 and CBS 115860, respectively.

In vitro susceptibility was tested according to the guidelines presented in document M38-A2 of the Clinical and Laboratory Standards Institute (CLSI) [6]. The minimum inhibitory concentration (MIC) was determined visually as the concentration that resulted in 100\% inhibition [6]. The minimal effective concentration (MEC) endpoint was taken as the lowest concentration at which the visual growth pattern change from granular to filamentous growth was detected, microscopically seen as restricted hyphal growth. The results revealed that multiple antifungal agents were inactive against the isolate, with MIC value of $4 \mu \mathrm{g} / \mathrm{ml}$ for voriconazole (VRC), $8 \mu \mathrm{g} / \mathrm{ml}$ for caspofungin (CAS), $>64 \mu \mathrm{g} / \mathrm{ml}$ for fluconazole (FLZ), $>16 \mu \mathrm{g} / \mathrm{ml}$ for itraconazole (ITC), posaconazole (POS), amphotericin B (AMB) and terbinafine (TRB), and MEC value of $>16 \mu \mathrm{g} /$ $\mathrm{ml}$ for micafungin (MCF). Due to the patient's economic condition, itraconazole was applied. However, despite the discouraging result of in vitro susceptibilities, significant improvement of skin lesions was achieved after 10 weeks treatment with itraconazole $200 \mathrm{mg}$ per day (Fig. 1c). No adverse event was reported.

\section{Discussion and conclusions}

M. cirrosus, the teleomorph of Scopulariopsis spp., is a rare opportunistic fungus and commonly found in soil and decaying organic matter. Up to date, there are only six cases of $M$. cirrosus infections reported, including three cases of systemic infection [2, 3, 5], two case of onychomycosis [4] and one case of primary cutaneous infection (the present case) (summarized in Table 1). Patients with systemic infection all had underlying disease that compromise the immune system. The present case represents the first primary cutaneous infection of $M$. cirrosus in immunocompetent individual. The development of cutaneous infection of this patient may be associated with the rural area that she lives in. Despite she denied any evident history of trauma, outdoor activities might provide opportunity of minor wounds and inoculation of the 
Table 1 Cases with M. cirrosus infection

\begin{tabular}{lllllllll}
\hline No & Year & Sex & Age & Underlying disease & Involved sites & Treatment & Outcome \\
\hline 1 & 1992 & F & 56 & - & Toenail & GSF, KTZ; Topical IMZ; & Resistant \\
& & F & 63 & - & Toenail & GSF; Topical MCZ & Resistant \\
2 & 1995 & M & 12 & AML, BMT & Skin, Lung & AMB & $\begin{array}{l}\text { Improvement in infection; Death } \\
\text { due to AML relapse }\end{array}$ \\
\hline 3 & 2006 & M & 49 & AML, BMT & Lung & AMB, VRC, TRB; Surgery; & Improvement in infection. \\
4 & 2018 & F & 60 & Emphysema SOT & Tracheo-bronchia & AMB, VRC, TRB, CAS, Debridement & Cure & [3] \\
5 & 2018 & F & 17 & - & Skin & ITC & Cure & - \\
\hline
\end{tabular}

Legend: AML Acute myelocytic leukemia, BMT Bone marrow transplantation, SOT Solid organ transplantation, GSF griseofulvin, KTZ ketoconazole, IMZ imidazole, $M C Z$ miconazole

pathogen. In addition, we suspected that the treatment with corticosteroids for Henoch-Sch nlein purpura might had transiently suppressed the immunity, resulting in the persistent infection.

Due to the rarity of the infection, the appropriate antifungal regimen for $M$. cirrosus infection has yet to be defined. Early cases of toenail infections showed resistant to systemic treatment with griseofulvin (GSF), ketoconazole (KTZ), and topical application of imidazole (IMZ), miconazole (MCZ) [4]. However, recent cases with systemic $M$. cirrosus infection all showed improvement with debridement and antifungal chemotherapy of amphotericin B alone or combined with voriconazole, terbinafine, and caspofungin, as shown in Table 1 [2, 3, 5]. Although in vitro susceptibility testing in this case revealed that $M$. cirrosus was resistant to a variety of antifungals, the patient achieved favorable effects with itraconazole, suggesting that there is an inconsistency between in vitro susceptibilities and in vivo antifungal effects.

In conclusion, although infection with $M$. cirrosus is a rare event, clinicians and microbiologists should be aware of the potential of $M$. cirrosus infection in both immunocompromised and immunocompetent individuals. Thorough mycological examination helps to recognize this rare pathogenic fungi, achieve an accurate diagnosis and initiate prompt antifungal treatments.

\section{Abbreviations \\ AMB: Amphotericin B; AML: Acute myelocytic leukemia; BMT: BMT Bone marrow transplantation; CAS: Caspofungin; CLSI: Clinical and Laboratory Standards Institute; FLZ: Fluconazole; GSF: Griseofulvin; IMZ: Imidazole; ITC: Itraconazole; ITS: Internal transcribed spacer; KTZ: Ketoconazole; MCF: Micafungin; MCZ: Miconazole; MEC: Minimal effective concentration; MIC: Minimum inhibitory concentration; POS: Posaconazole; SDA: Sabouraud's dextrose agar; SOT: Solid organ; TRB: Terbinafine; VRC: Voriconazole}

\section{Acknowledgements}

We thank Professor Ruoyu Li, Professor Jin Yu, Zhe Wan and Yabin Zhou from Peking University First Hospital, Research Center for Medical Mycology, Peking University, Beijing, China for pathologic diagnosis assistance.

\section{Funding}

This report received no specific grant from any funding agency in the public, commercial, or not-for-profit sectors.
Availability of data and materials

All data generated or analyzed during this study are included in this published article. The sequence data have been deposited in the GenBank database (http://www.ncbi.nlm.nih.gov/Genbank/index.html) with the accession number MF450505 and MF455507.

\section{Authors' contributions}

DG and JC collected and interpreted the clinical data, and wrote the manuscript. LG designed, interpreted the clinical data and wrote the manuscript. ML revised the manuscript critically for important content. LG carried out the microbiological examination and nucleotide sequencing. All authors read and approved the final manuscript.

\section{Ethics approval and consent to participate}

Due approval from the Institutional Research and Ethics Committee of Zhongshan Hospital Fudan University was obtained for analysing the case study and writing the manuscript.

\section{Consent for publication}

Written informed consent was obtained from the parents of the patient described in this report. A copy of the written consent is available by request.

\section{Competing interests}

The authors declare that they have no competing interests.

\section{Publisher's Note}

Springer Nature remains neutral with regard to jurisdictional claims in published maps and institutional affiliations.

Received: 20 July 2018 Accepted: 20 November 2018

Published online: 03 December 2018

References

1. Sandoval-Denis M, Gene J, Sutton DA, Cano-Lira JF, de Hoog GS, Decock CA, Wiederhold NP, Guarro J. Redefining Microascus, Scopulariopsis and allied genera. Persoonia. 2016;36:1-36.

2. Krisher KK, Holdridge NB, Mustafa MM, Rinaldi MG, McGough DA. Disseminated Microascus cirrosus infection in pediatric bone marrow transplant recipient. J Clin Microbiol. 1995:33(3):735-7.

3. Ustun C, Huls G, Stewart M, Marr KA. Resistant Microascus cirrosus pneumonia can be treated with a combination of surgery, multiple antifungal agents and a growth factor. Mycopathologia. 2006;162(4):299-302.

4. Devroey C, Lasagni A, Tosi E, Schroeder F, Song M. Onychomycoses due to Microascus-Cirrosus (Syn M-Desmosporus). Mycoses. 1992;35(7-8):193-6.

5. Taton O, Bernier B, Etienne I, Bondue B, Lecomte S, Knoop C, Jacob F, Montesinos I. Necrotizing Microascus tracheobronchitis in a bilateral lung transplant recipient. Transpl Infect Dis. 2018;20:e12806.

6. Clinical and Laboratory Standards Institute. Reference method for broth dilution antifungal susceptibility testing of filamentous fungi; approved standard-2nd ed. CLSI document M38-A2. Wayne, PA: CLSI; 2008. 